Chemical Engineering Science, 1969, Vol. 24,pp. 1043-1054. Pergamon Press. Printed in Great Britain.

\title{
The influence of reacting gases on the motion of collapsing cavities
}

\author{
SCOTT FOGLER \\ Department of Chemical and Metallurgical Engineering, University of Michigan, Ann Arbor, Mich. \\ 48104, U.S.A.
}

(First received 16 A ugust 1968; in revised form 6 February 1969)

\begin{abstract}
An analysis was performed on a collapsing cavity containing reacting gases. It was determined that with reacting gases in the cavity there was significant departure from the fluid mechanics of collapse of a cavity containing inert gases. When reacting gases are present, the nonlinear differential equations of motion, energy, and kinetics must be solved simultaneously. It was found that the time required to reach a given radius during collapse was greater for an exothermic reaction occurring in the gas phase than for the cavity containing a nonreacting gas. For the exothermic reaction the collapse time increases with increasing magnitude of the heat of reaction, while for an endothermic reaction the collapse time does not increase indefinitely with increasing heat of reaction but instead decreases and approaches a finite limit. For small heats of reaction the influence of a reacting gas on cavity motion is only readily detectable during the rebound of the cavity. When the collapse time approaches the half period of the wave, the conversion increases with increasing acoustic pressure. However, when the collapse time approaches the period of the wave, the conversion first decreases, then increases with increasing acoustic pressure. Additional descriptions are presented in the text delineating the behavior of the cavity during the collapse when a gas is reacting within the cavity.
\end{abstract}

\section{INTRODUCTION}

IN 1754 Leonhard Euler postulated that small voids would occur in a liquid subjected to a small negative pressure at a point. It was not until 150 yr later that these voids were observed. The first observances of these bubbles occurred on ship's propellers, and the name given by $\mathbf{R}$. E. Froude to the phenomenon associated with these bubbles was "cavitation". The term is now taken to mean the nucleation, growth, and collapse of gas and vaporous bubbles and voids in liquids. In an effort to describe the eroding action of these bubbles, Rayleigh [1] was the first to develop a mathematical model of the collapsing bubble. In addition to solving the collapse equations for a spherical void, he found that for a gas filled cavity the contents of the cavity were compressed to very high pressures in the final stages of collapse. The shock produced by this collapsing bubble hitting the metal surface was believed responsible for producing the observed erosion.

The equations of motion describing the collapse of the bubble put forth initially by Rayleigh were later modified and extended by Kirkwood [2], Trilling [3], Plesset [4-6], Flynn [7], and others. In the early 1950's, Noltingk and Neppiras [8,9] studied the problem of cavitation produced by ultrasonic waves. They postulated the following account of a cavitation bubble. During the rarefaction phase of the wave, nuclei within the liquid are attacked and small bubbles are formed at these points. As the rarefaction phase of the wave proceeds to act on the newly formed cavity, the bubble begins to grow. As the cavity grows, the liquid at the gasliquid interface inside the cavity evaporates into the cavity. Also during this time, gases which are dissolved in the liquid can diffuse into the cavity. The cavity continues to grow to some maximum radius until the condensation phase of the wave begins to act on the cavity. The collapse of the cavity is initiated, and the contents of the cavity are compressed to very high temperatures and pressures during the collapse phase. These high temperatures and pressures are believed responsible for the observed erosion, luminescence, and increased chemical reaction rates 
brought about by the application of ultrasonic waves to liquid phase systems. Numerous reaction yields are greatly increased with this application. In some instances certain reactions, such as the carbontetrachloride-water reaction, do not proceed to any measurable extent without the application of ultrasonic cavitation. This work concerns the fundamental study of a reacting gas contained in the cavitation during collapse and the effects of the reacting gas on the fluid mechanics of the collapse.

\section{KINETIC EQUATIONS}

In this study, discussion will be limited to single step elementary reactions which follow an Arrhenius rate law. For a reaction involving $N$ chemical species, $q$ of which are products and $N-q$ of which are reactants, the overall equation can be written as

$$
\sum_{i=1}^{N} \nu_{i} A_{i}=0
$$

where $A_{i}$ is the $i$ th species and $\nu_{i}$ is the stoichiometric coefficient of the $i$ th species. The reaction rate, $r$, per unit volume (e.g. g moles/cc/sec) for an elementary reaction is given by

$$
r=k_{1}\left\{\prod_{i=1}^{q} C_{i}^{o_{1}}-\frac{\prod_{i=q+1}^{N} C_{i}^{o_{i}}}{K_{c}}\right\} .
$$

The quantity $k_{1}$ represents the forward specific reaction rate constants while $C_{i}$ is the concentration of species $i$. The temperature dependence of the specific reaction rate constant, $k$, is given by the Arrhenius equation.

$$
k=f \mathrm{e}^{-E / B T} .
$$

The value of the concentration equilibrium constant at any temperature, $T$, is

$$
K_{c}(T)=\left[K_{p}\left(T_{0}\right)(B T)^{-\Sigma \nu_{i}}\right] \exp \int_{T_{0}}^{T} \frac{\Delta H}{B T^{2}} \mathrm{~d} T
$$

where the heat of reaction is a function of temperature.

\section{CONSERVATION OF MASS}

The concentration, $C_{i}$, can be expressed in terms of the total volume of the cavity, $V$, and the number of moles of component $i, n_{i}$, i.e. $C_{i}=n_{i} / V$. The number of moles of component $i$ can be obtained from a mass balance in which there is no transfer of mass across the cavity wall. The rate of accumulation of component $i$ is then equated to the rate of chemical generation of species $i$.

$$
\frac{\mathrm{d} n_{i}}{\mathrm{~d} t}=\nu_{i} r V
$$

The overall mass balance gives the total number of moles in the cavity at any time $t$ as

$$
n=n_{0}+\Delta \nu \int_{0}^{t} r v \mathrm{~d} t .
$$

Using $X$ to represent the number of moles of species 1 that have reacted per total mole initially, Eq. (5) can also be written in the form:

$$
n_{0} \frac{\mathrm{d} X}{\mathrm{~d} t}=r V .
$$

Letting $\beta_{i}$ represent the mole fraction of $i$ initially, the number of moles of species $i$ at any time $t$ is

$$
n_{i}=n_{0}\left(\beta_{i}+\nu_{i} X\right) .
$$

In order to solve Eq. (7) and thus determine the moles reacted as a function of time and cavity radius, the above kinetic equation must be coupled with the energy equation and the momentum equation, as the cavity temperature and volume must be known functions of time. The volume-time relationship is obtained from the momentum equation.

\section{EQUATIONS OF MOTION}

This study is concerned with reacting gases during the collapse of a cavity in a homogeneous liquid under the influence of an ultrasonic wave. Various models describing the motion of a collapsing cavity have been developed and these 
models can be grouped into two categories. One category contains those models which treat the liquid as being compressible, and the other contains those in which it is treated incompressible. With a division of this type, one can write a general equation for each category and show how the various mathematical descriptions, such as those of Guth [10], Noltingk [8], and Khoroshev [11], as well as of this work, can be deduced from this one general equation.

The development of the general equation in the case of an incompressible liquid is a modification of the equations for bubble growth without chemical reaction given by Scriven $[12,13]$. In the liquid phase outside the cavity wall the continuity equation for a homogeneous incompressible liquid is expressed in spherical coordinates as

$$
\frac{\partial}{\partial r_{1}}\left(r_{1}^{2} u\right)=0
$$

Assuming that the ratio of the density of gas inside the bubble to the liquid density is small and letting $R$ and $R$ represent the radius of the cavity and the velocity of the cavity wall respectively, Eq. (9) can be integrated to give

$$
u r_{1}^{2}=g(t)=R^{2} \stackrel{\circ}{R} .
$$

In the absence of body force and external temperature effects, and considering the fluid motion to be in the laminar flow regime, the momentum balance is

$$
\rho\left(\frac{\partial u}{\partial t}+u \frac{\partial u}{\partial r_{1}}\right)=-\frac{\partial P}{\partial r_{1}}-|\nabla \cdot \bar{\tau}|_{r_{1}} .
$$

Dividing by liquid density, $\rho$, substituting for $u$ in terms of $R$ and $\stackrel{R}{R}$ in Eq. (11), and then integrating between $r_{1}=R$ and $r_{1} \rightarrow \infty$ at a particular time gives the dynamic equation governing the collapse of the cavity.

$$
R \stackrel{\circ}{R}+\frac{3}{2} \dot{R}^{2}=\frac{P_{l}(R)-P_{\infty}(\infty)}{\rho}-\frac{1}{\rho} \int_{R}^{\infty}|\nabla \cdot \overline{=}|_{r_{1}} \mathrm{~d} r_{1} .
$$

If the fluid is Newtonian, the last term in Eq. (14) involving the stress tensor, $\overline{\bar{\tau}}$, is zero.

The pressure a large distance from the cavity wall, $\boldsymbol{P}_{\infty}$, will in some instances be a function of time. The pressure on the liquid side of the cavity wall, $\boldsymbol{P}_{l}$, is obtained from a momentum balance at the cavity gas-liquid interface which requires:

$$
P_{l}(R)+\tau_{r r, l}+\frac{2 \sigma}{R}=P_{g}+\tau_{r r, g} .
$$

The surface tension is denoted by $\sigma ; P_{g}$ is the gas pressure inside the cavity; while the subscripts $g$ and $l$ refer to the gas and the liquid, respectively. Substitution of Eq. (13) into Eq. (12) gives the following expression from which the various mathematical descriptions of a collapsing cavity can be obtained.

$R \stackrel{\infty}{R}+\frac{3}{2} \stackrel{\circ}{R}^{2}=\frac{P_{g}(R)-P(\infty)}{\rho}-\frac{2 \sigma}{R \rho}+\frac{\tau_{r r, g}-\tau_{r r, l}}{\rho}$.

The normal stress is

$$
\tau_{r r}=-2 \mu \frac{\partial u}{\partial r}+\left(\frac{2}{3} \mu+\kappa\right) \nabla \cdot \mathbf{V},
$$

where $\kappa$ is the dilational or bulk coefficient of viscosity and $\mu$ is the shear coefficient of viscosity. Since the liquid is considered incompressible, the divergence of the liquid velocity vector $V_{l}$, is zero and the stress becomes:

$$
\tau_{r r, l}=-2 \mu\left(\frac{\partial u}{\partial r}\right)_{r=R}=4 \mu\left(\frac{\stackrel{\circ}{R}}{R}\right) .
$$

For no flow of material in or out of the gas cavity, the overall mass balance is:

$$
\rho_{g} R^{3}=\text { const. }
$$

differentiating with respect to time and rearranging:

$$
-\frac{\mathrm{d} \ln \rho_{g}}{\mathrm{~d} t}=3 \frac{\stackrel{\circ}{R}}{R}
$$




\section{S. FOGLER}

Neglecting any spatial variation in the gas density, $\rho_{g}$, inside the cavity, the gas side continuity equation is

$$
-\frac{\mathrm{d} \ln \rho_{g}}{\mathrm{~d} t}=+\frac{1}{r_{1}^{2}} \frac{\partial}{\partial r_{1}}\left(r_{1}^{2} u_{g}\right) .
$$

The gas phase velocity gradient at the cavity radius $R$ is found by Eqs. (18) and (19)

$$
\left(\frac{\partial u_{g}}{\partial r_{1}}\right)_{g, R}=\frac{\stackrel{\circ}{R}}{R}
$$

The normal stress, $\tau_{r r, b}$ at $r_{1}=R$ is

$$
\tau_{r r, g}=-3 \kappa \frac{\stackrel{\circ}{R}}{R}
$$

When Eqs. (16) and (21) are inserted into Eq. (14), the following equation is obtained:

$\stackrel{\infty}{R} R+\frac{3}{2} \stackrel{\circ}{R}^{2}=\frac{P_{g}(R)-P_{\infty}(t)}{\rho}-\frac{2 \sigma}{R \rho}-\frac{\stackrel{R}{R}}{R} \cdot \frac{\left(4 \mu_{l}+3 \kappa\right)}{\rho}$.

For ideal gases the dilational viscosity is zero [Liebermann, 14], and for small departures from ideality at moderate pressures, it is the same order of magnitude as the shear coefficient of viscosity, $\mu_{g}$, and can be neglected in comparison with the liquid phase viscosity, $\mu$.

Under these circumstances the momentum equation is:

$$
\stackrel{\infty}{R} R+\frac{3}{2} \stackrel{\circ}{R}^{2}+\frac{4 \mu}{R} \stackrel{\therefore}{R}=\frac{P_{g}(R)-P_{\infty}}{\rho}-\frac{2 \sigma}{R \rho} .
$$

If the heat of reaction and stoichiometric volume change are small, the reacting gases in the cavity have a negligible effect on the equation of motion. As a result, once the solution of Eq. (23), i.e. $R(t)$, is obtained for an inviscid gas, the cavity temperature and volume at any time, $t$, can be quickly calculated for use in the kinetic equations. However, for large heats of reaction and stoichiometric volume changes, Eq. (23) must be coupled with the differential energy equation for a solution to be obtained.
The equations of Noltingk and Neppiras [9], can be obtained from the general equation (i.e. Eq. 23) by neglecting both the liquid and gas viscosities and letting the pressure a large distance from the cavity vary as the superposition of the acoustic pressure, $P_{a}$, and atmospheric pressure, $P_{0}$, vary.

$$
P_{\infty}=P_{0}+P_{a} \sin (\omega t) .
$$

The pressure, $P_{g}$, given by Noltingk and Neppiras at some other radius $(R)$ is given by

$$
P_{g}(R)=P_{g}\left(R_{0}\right)\left(\frac{R_{0}}{R}\right)^{3}
$$

In other words, they have assumed the gas to be compressed isothermally.

Guth, in deriving his formula for the minimum radius reached upon collapse, used the adiabatic pressure-volume relationship rather than the isothermal relationship. This work will not be restricted by the gas pressure equations used by either Guth or Noltingk. Consequently the equation for the cavity radius is given by

$$
\stackrel{\infty}{R} R+\frac{3}{2} R^{2}+\frac{2 \sigma}{\rho R}=\frac{P_{g}-\left(P_{0}+P_{a} \sin (\omega t)\right)}{\rho}-\frac{4 \mu}{\rho} \frac{\stackrel{\circ}{R}}{R} .
$$

In the case of no chemical reactions and no mass flow out of the cavity, the gas pressure is given by either the adiabatic or isothermal pressure volume relationship. When a chemical reaction is occurring in the collapsing cavity, $P_{g}$ must be obtained from the energy equation and equation of state.

\section{CONSERVATION OF ENERGY}

Taking the gas phase as the system, letting $q$ be the heat flow rate to the cavity and $W$ be the work done by the system on the surroundings, the energy balance on the system is readily obtained from the first law of thermodynamics. From previous studies on a collapsing cavity, it is known that the temperatures obtained during the final stages of collapse are quite high. There- 
fore the ideal gas assumption is quite good, and consequently, the heat of mixing is zero and the partial molal enthalpies are set equal to the enthalpies of the pure components. Using the differential definition of enthalpy, $H$, by dividing the work into $P V$ work and shear work and substituting into the energy balance, the result is

$$
q \mathrm{~d} t-\mathrm{d} W_{\tau}=\mathrm{d} H-V \mathrm{~d} P .
$$

The total enthalpy of the system can be expressed in terms of the individual species $i$. Differentiating the total enthalpy and then substituting form $\mathrm{d} n_{i}$ obtained from the mole balance, one can obtain the energy equation in the form

$$
q \mathrm{~d} t+V \mathrm{~d} P-\mathrm{d} W_{\tau}=\sum n_{i} C_{p_{i}} \mathrm{~d} T+\Delta H r V \mathrm{~d} t .
$$

The differential shear work, $\mathrm{d} W_{\tau}$ is

$$
\mathrm{d} W_{\tau}=\tau_{r r, g} A \mathrm{~d} R=-12 \pi \kappa_{g} \stackrel{\circ}{R}^{2} R \mathrm{~d} t .
$$

The term $V \mathrm{~d} P$ is obtained by differentiating the ideal gas law, and $\mathrm{d} n$, the change in the total number of moles, is obtained by differentiation of Eq. (6). Utilizing these relationships and upon substitution of Eq. (29) into Eq. (27), the energy equation reduces to

$$
\begin{aligned}
q+ & 12 \pi \kappa \stackrel{\circ}{R}^{2} R-n B T \frac{\mathrm{d} \ln V}{\mathrm{~d} t} \\
& =\left(\Sigma n_{i} C_{p i}-n B\right) \frac{\mathrm{d} T}{\mathrm{~d} t}+r V(\Delta H-\Delta \nu B T) .
\end{aligned}
$$

As previously mentioned, $\kappa$ is zero for an ideal gas, and the cavity collapse will be taken to be adiabatic. Since a large portion of this work is concerned with effects of chemical reactions on the collapse of the cavity, only those conditions which met criteria for cavities approaching adiabatic collapse were of interest. The criteria set forth by Jameson [15] for an adiabatic collapse were fulfilled in this work. With these considerations the energy equation is

$$
\frac{\mathrm{d} T}{\mathrm{~d} t}=\frac{((\Delta \nu B T-\Delta H) r V-n B T \mathrm{~d} \ln V / \mathrm{d} t)}{\Sigma n_{i} C_{P_{i}}-n B} .
$$

In order to obtain a solution, the energy balance, Eq. (31), must be coupled with the momentum balance, Eq. (26),

$$
R \ddot{R}+\frac{3}{2} \dot{R}^{2}+\frac{2 \sigma}{\rho \dot{R}}=\frac{P_{g}-\left(P_{0}+P_{a}(\sin \omega t)\right)}{\rho}-\frac{4 \mu \stackrel{R}{R \rho}}{R},
$$

the equation of state,

$$
P_{g}=\frac{n B T}{V}
$$

the kinetic equation,

$$
\begin{aligned}
n_{0} \frac{\mathrm{d} x}{\mathrm{~d} t}=r V=k_{1} V & {\left[\prod_{i=1}^{q}\left(\frac{n_{0}\left(\beta_{i}+\nu_{i} X\right)}{V}\right)^{0_{1}}\right.} \\
& \left.-\prod_{i=q+1}^{N} \frac{\left(\frac{n_{0}\left(\beta_{i}+\nu_{i} X_{1}\right)}{V}\right)^{0_{1}}}{K_{c}}\right]
\end{aligned}
$$

and the other equations previously defined which give the variation of the parameters, (e.g. $K_{c}(T)$ ), with one of the dependent variables, (e.g. T). The initial conditions for the solutions considered in this work are that at time $t=0$, then $R=R_{0}, \stackrel{R}{R}=0, n=n_{0}, T=T_{0}$ and $X=0$.

\section{DISCUSSION}

In the figures that follow, typical values of the kinetic parameters, activation energy and frequency factor, were taken from Smith [16], International Critical Tables [17], and Tables of Chemical Kinetics Data[18]. Corresponding values of the heat of reaction, equilibrium constant, and heat capacities were obtained from Hougen et al.[19] and JANAF [20]. Typical values of $n_{0}$ and $R_{0}$ were taken from Flynn [7] and Boguslavskii[21], and the remaining parameter values (e.g. $\sigma$ and $\mu$ ) were readily obtained from the Handbook of Chemistry and Physics [22]. The numerical integration program used in this research was an extension of Romberg's extrapolation algorithm and is presented for the most part by Bulirsche [23].

Figure 1 shows the radius-time curves for a Guth cavity with a specific heat ratio, $\gamma$, of 1.2 and for a Rayleigh cavity. The point $A$ on the 


\section{S. FOGLER}

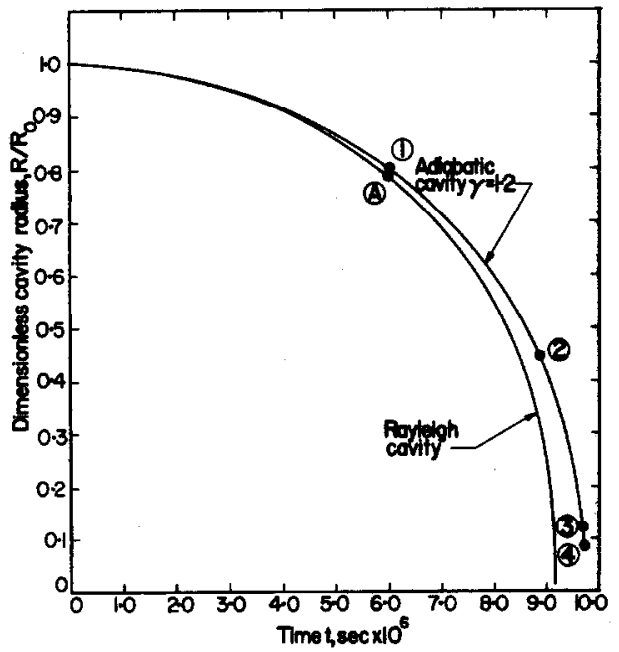

Fig. 1. Comparison of the radius-time collapse curves of a Guth and a Rayleigh cavity.

Rayleigh curve represents the radius at which the inertial force, $\frac{3}{2} \rho R^{2}$, and the pressure force, $P_{g}-P_{0}$, are equal and are acting in a direction to accelerate the collapse. At a radius greater than that at point $A$, the inertial force is the controlling factor. Point 1 on the adiabatic collapse curve also represents the point at which the inertial and pressure forces are equal. Between points 1 and 2 the inertial force increases while the pressure force decreases. This net decrease is due to the increase of the pressure inside the cavity resulting from the adiabatic compression of the cavity. At point 2 the gas pressure is equal to the external pressure on the cavity, and the net pressure force on the cavity is zero. Between points 2 and 3 the gas pressure inside the cavity, which is now greater than the external pressure, continues to increase until the pressure force is equal to the inertial force and acts in the opposite direction. This condition occurs at point 3 , where the inward acceleration term, $\boldsymbol{R} \boldsymbol{R}$, changes from negative to positive, and the velocity is a maximum. From points $3-4$ the velocity continues to decrease to zero, where the cavity obtains its minimum radius. The cavity begins to rebound at this point due to the large internal gas pressure acting to expand the cavity. If the cavity should become unstable and shatter in the final stages of compression, of course this rebound will not occur.

Figure 2 shows a comparison of the collapse curves for cavities in which either, (1) an exothermic reaction, (2) an endothermic reaction or

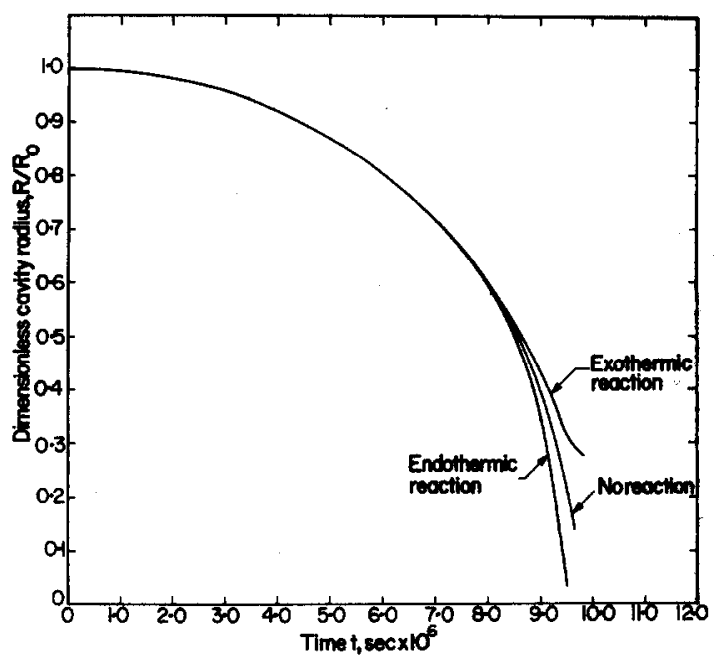

Fig. 2. Comparison of the collapse curves of a cavity in which there is either no reaction, an exothermic reaction, or an endothermic reaction.

(3) no reaction takes place during the collapse. As can be seen from this figure, the cavity containing the gas in which an exothermic reaction is taking place requires a longer time to reach the same radius in the final stages of collapse than the cavity in which no reaction is taking place. This results from the fact that energy is released by the reacting gases in addition to the energy from the adiabatic compression of the gas in the cavity. This release increases the gas temperature and pressure above the case of no reaction and retards the collapse to a greater extent than the collapse without reaction. Thus, as a result of the increased retardation, the cavity takes a longer period of time to reach the same radius as collapse without reaction. It is also noted that the minimum radius reached on colapse is greater for the exothermic case. When the reaction is endothermic, the compression energy is absorbed by the reaction, resulting in a lower gas temperature and pressure than that of 
the collapse where the gases do not react. Therefore the gas offers less resistance to collapse than in the non-reacting case. The time required to reach a given radius is less in the endothermic case than in either the exothermic or the nonreacting case.

If the heat of reaction is small, neither the endothermic nor the exothermic collapse can be easily distinguished from collapse of the cavity containing no reacting gases. It is only on the rebound that endothermic and exothermic reactions can be distinguished. The exothermic reaction cavity rebounds to a radius higher than the non-reacting cavity, while the endothermic cavity rebounds to a radius below the nonreacting cavity. This result is shown in Fig. 3. It should be noted that for a reaction to have a pronounced effect on the collapse, the specific reaction rate constant must be large enough so that the heat of reaction will be released at a sufficient rate to affect the collapse.

When the magnitude of the heat of reaction is allowed to increase indefinitely, the following bounds are found to exist on the collapse. Since the gas pressure increases with increasing exothermic heat of reaction, the minimum radius

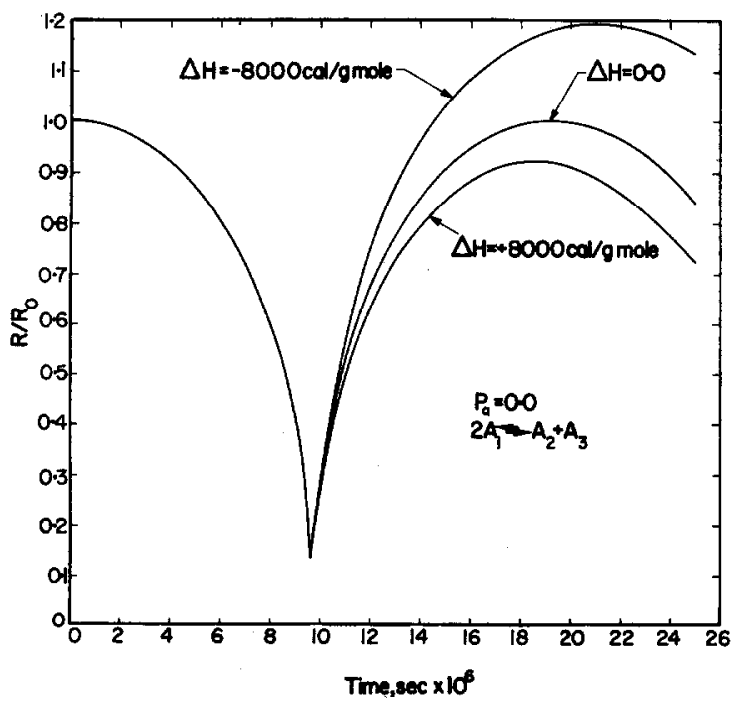

Fig. 3. The collapse and rebound of a cavity in which there is either a small exothermic, a small endothermic, or no heat of reaction release. increases with increasing exothermic $\Delta H$ until the initial radius is reached. Any further increase in the magnitude of $\Delta H$ beyond this point would cause the cavity to grow rather than collapse. Therefore, as the heat of reaction increases without bound, the cavity radius and collapse time increase without bound. For an endothermic reaction, the collapse time does not increase indefinitely with increasing $\Delta H$, but instead decreases and approaches a finite value. The radius time curve for the endothermic reaction approaches the radius time curve of that of a Rayleigh collapse. If the liquid viscosity and surface tension are neglected, the collapse time, $\tau$, approaches the Rayleigh collapse time, $\tau_{R}$.

$$
\lim _{\Delta H \rightarrow \infty} \tau=\tau_{R}=0.915 R_{0} \sqrt{\left(\frac{\rho_{0}}{P_{0}}\right)}
$$

The conversion-radius curves in the absence of an acoustic wave are given in Fig. 4 for four different activation energies. The conversion, $x$, shown in this figure, is for an irreversible second order exothermic reaction. As can be seen in this plot, most of the product is formed within a very small change in cavity radius, $\Delta R$. For an activation energy of $20 \mathrm{kcal} / \mathrm{g}$ mole, $95 \mathrm{per}$ cent of the reaction takes place within a $\Delta R / R_{0}$ of

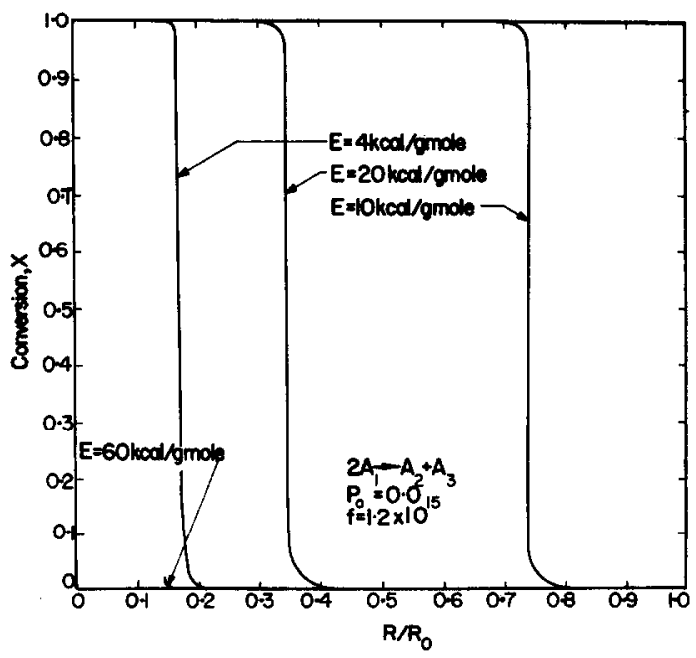

Fig. 4. Conversion for an irreversible second order reaction as a function of radius when no acoustic wave is present. 


\section{S. FOGLER}

0.01 . This change in radius, $\Delta R / R_{0}$, at which a substantial degree of conversion is obtained, decreases with increasing heat of reaction for a given activation energy. When the reaction is. endothermic or there is heat conduction out of the cavity, the conversion-radius curves are not nearly so steep as those shown. The increase in $\Delta R / R_{0}$ in these instances is due to reduction in the reaction rate resulting from lower cavity temperatures.

The radius at which the reaction rate reaches its maximum value decreases with increasing activation energy. This is due to the fact that higher temperatures, and thus smaller radii, are necessary for the reaction to proceed at its maximum rate. For the case of the reaction with an activation energy of $60 \mathrm{kcal} / \mathrm{g}$ mole, only 1.5 per cent conversion was obtained by the time the cavity reached its minimum radius.

Figure 5 shows similar curves for activation energies of 60,40 , and $20 \mathrm{kcal} / \mathrm{g}$ mole when an acoustic pressure of 0.4 bars is acting on the cavity. The only significant difference between the conditions of Figs. 4 and 5 is the degree of conversion accomplished for an activation energy of $60 \mathrm{kcal} / \mathrm{g}$ mole. Comparing the two figures, it is easily seen that the conversion increases from 1.5 per cent to 97 per cent when

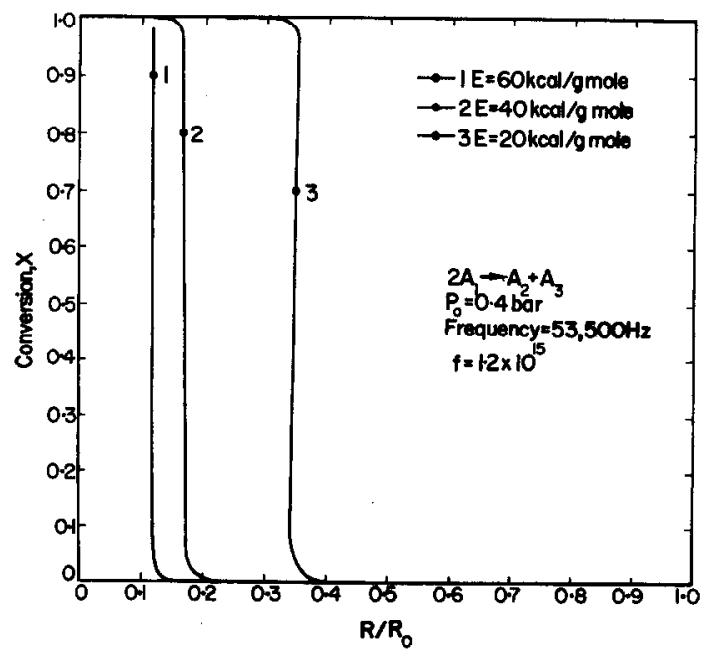

Fig. 5. Conversion for an irreversible second order reaction as a function of radius in the presence of an acoustic wave. the acoustic pressure is changed from zero to 0.4 bars.

Next consider the class of cavities with radius $R_{0}$ and corresponding collapse time $\tau$ under the influence of an acoustic wave of period $T$ for which

$$
\lim _{P_{a} \rightarrow 0}\left\{\frac{\tau}{T}\right\}=1
$$

It is interesting to consider what happens to the conversion $X$, obtained at the minimum radius of collapse as the acoustic pressure for this class of cavities is increased from zero to 1 bar. Figure 6 shows this variation in conversion with acoustic pressure and the corresponding

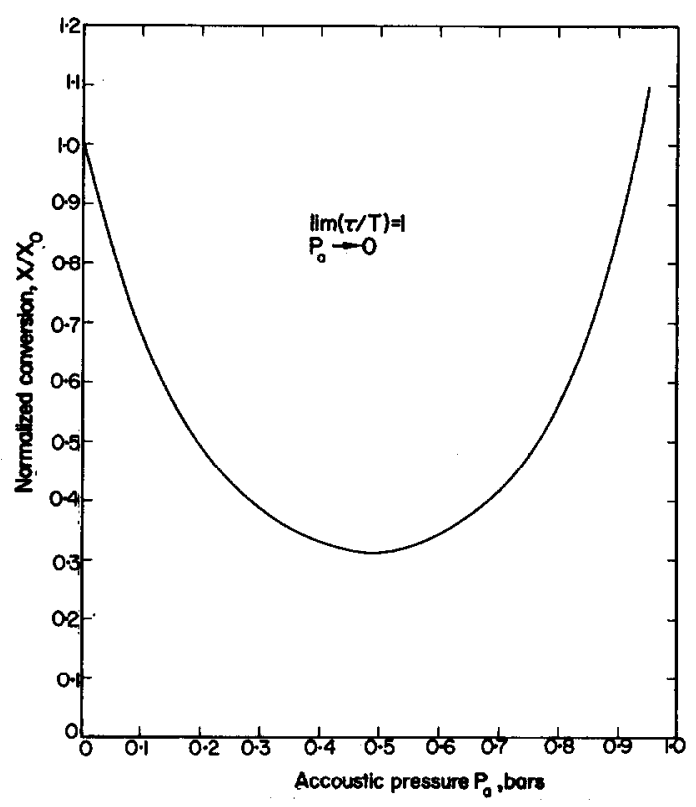

Fig. 6. Variation of conversion with acoustic pressure in a second order reaction for the class of cavities in which the collapse time approaches the period of the wave at low acoustic pressures.

minimum in the conversion curve. For small acoustic pressures, the intensity of collapse is reduced below that without the application of the wave, and as a result, the conversion is less. The occurrence of this minimum can best be understood by considering the relative magnitude of the two dominant forces (inertial, $\frac{3}{2} \stackrel{\circ}{R}^{2} \rho$, 
and pressure, $\left.P_{g}-\left(P_{0}+P_{a} \sin \omega t\right)\right)$ involved during the various stages of collapse.

In the period of collapse, $T / 2 \geqslant t>0$, the acoustic pressure is positive and tends to accelerate the collapse with increasing acoustic pressure. During this period the inertial forces increase with increasing acoustic pressure. On the other hand, the acoustic pressure is negative during the time, $T>t>\mathrm{T} / 2$, and decelerates the inward motion. At the time the rarefaction phase begins to act on the cavity, $t=T / 2$, the pressure forces are dominant for small acoustic pressures. Under these conditions, the rarefaction phase of the wave will have a pronounced effect since the pressure forces are controlling. For acoustic pressures approaching 1 bar, the inertial forces are controlling at $t=T / 2$, the time at which the rarefaction phase of the wave begins acting on the cavity. Consequently, the retardation effects of the wave during the second half of the cycle are overshadowed by the increase in inertial force conceived during the first half of the cycle. The intensity of collapse is therefore increased, resulting in an increase to, and in some cases even above, the level obtained in the absence of the wave. When $t=T / 2$, it is not surprising that the conversion is at its minimum when the ratio of inertial to pressure forces is nearly unity. For an acoustic pressure, $P_{a}$, below the point where the conversion is a minimum, this ratio is less than unity, while the ratio is greater than unity for a $P_{a}$ above the minimum conversion.

Figure 7 shows the variation of the natural $\log$ of conversion with acoustic pressure for two second order specific reaction rate constants. The wave frequency was chosen such that the collapse time approached one-half period of the wave for exceedingly small acoustic pressures. Under these conditions only the condensation phase of the wave acts upon the cavity during the collapse, and as a result, the degree of compression and the intensity of collapse increase with increasing acoustic pressure. When the conversion is greater than 5 per cent, it increases very rapidly with acoustic pressure. At this level of conversion, the exothermic heat

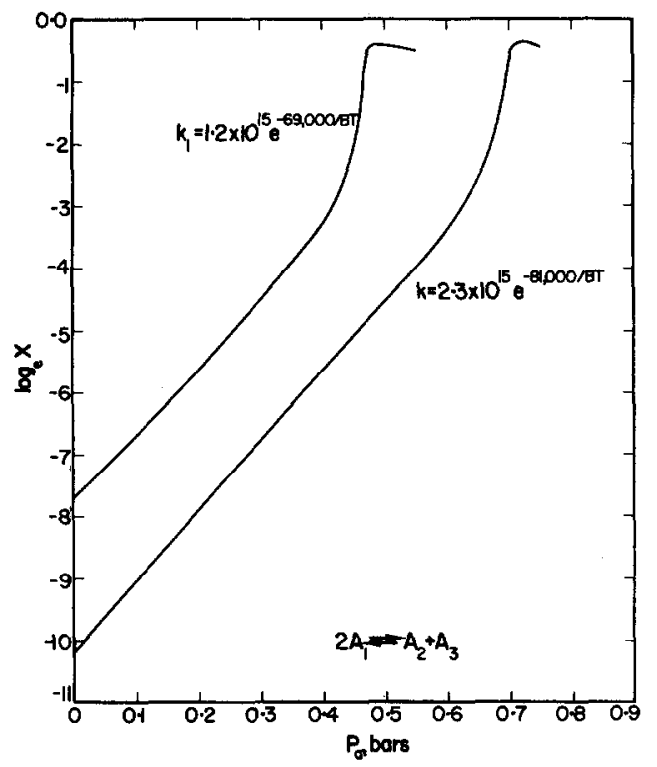

Fig. 7. Variation of conversion with acoustic pressure of a second order reversible reaction for the class of cavities in which the collapse time approaches the half period of the wave at low acoustic pressures.

reaction begins to add significantly to the temperature rise resulting from the adiabatic compression. This increased temperature in turn increases the reaction rate and the conversion, which further increases the exothermic heat of reaction release. This thermal catalytic process continues in this manner until the equilibrium conversion is reached. Increasing $\boldsymbol{P}_{\boldsymbol{a}}$ beyond the point where equilibrium is first reached will continue to increase the temperature in the cavity at the minimum radius. This increased temperature will in turn cause the equilibrium to shift the exothermic reaction in the reverse direction, resulting in decreasing conversion with further increases in acoustic pressure. It is also seen in this figure that higher acoustic pressures are necessary to reach the equilibrium conversion for higher activation energies.

Figure 8 shows the conversion-time curve for reversible second order reaction which reaches equilibrium before reaching its minimum radius. At time $t=0$, the cavity collapse begins. The reaction equilibrium point is reached at $9.25 \mu$ 


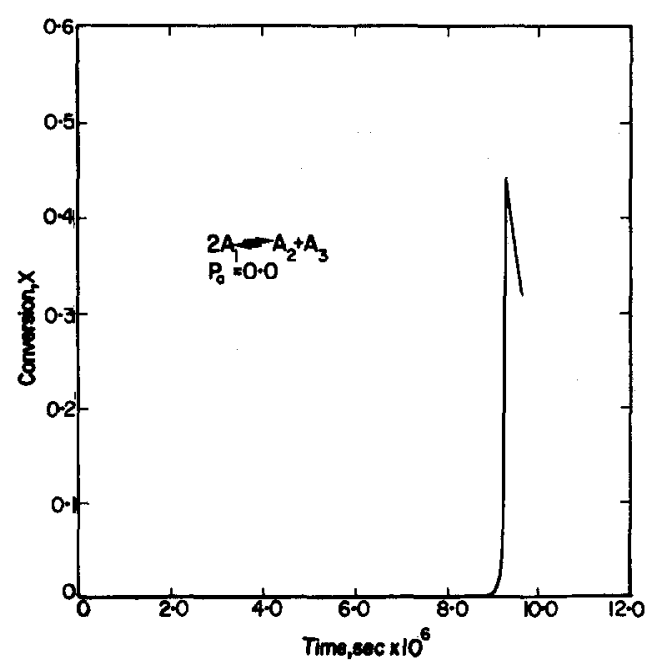

Fig. 8. Conversion for a second order reversible reaction as a function of time during the collapse.

sec, and the cavity reaches its minimum radius after $9.66 \mu \mathrm{sec}$.

During the time between 9.25 and $9.66 \mu \mathrm{sec}$, the reverse reaction rate is greater than the forward rate, and the reaction products are converted back into reactants. Since the temperature is continually increasing, the equilibrium constant continues to decrease, shifting the reaction even further in the reverse direction. At $t=9.66$, the temperature is at its maximum value. If the cavity rebounds, the temperature will decrease owing to the adiabatic expansion of the gas. As the temperature decreases during rebound, the equilibrium constant increases. The reaction again proceeds in the forward direction increasing the conversion. The phase plot illustrating this occurrence is given for the rebounding cavity in Fig. 9. The upper curve in this figure represents the conversion during rebound, while the lower one gives the conversion during collapse.

For large activation energies and small acoustic pressures, it was shown in Figs. 4 and 7 that very little conversion was obtained upon collapse of the cavity. Figure 10 shows how conversion continually increases with each rebound for an oscillating cavity under the influence of an acoustic pressure of 0.05 bars.

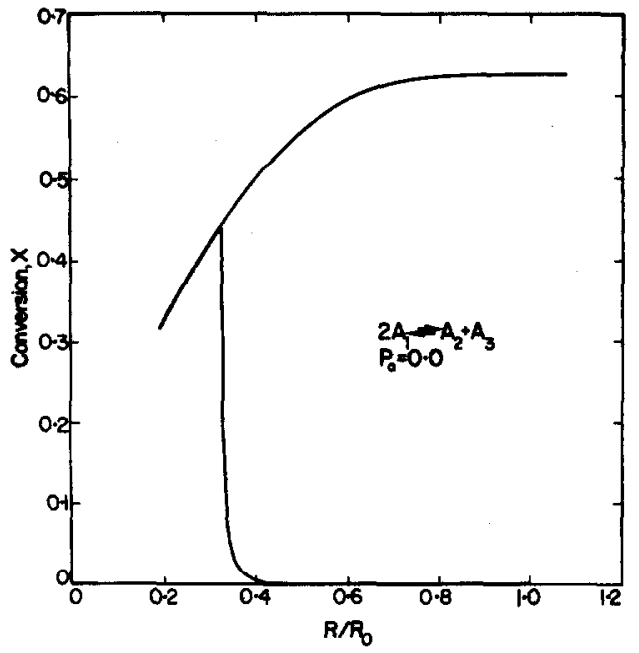

Fig. 9. Conversion for a second order reversible reaction as a function of radius during collapse and rebound.

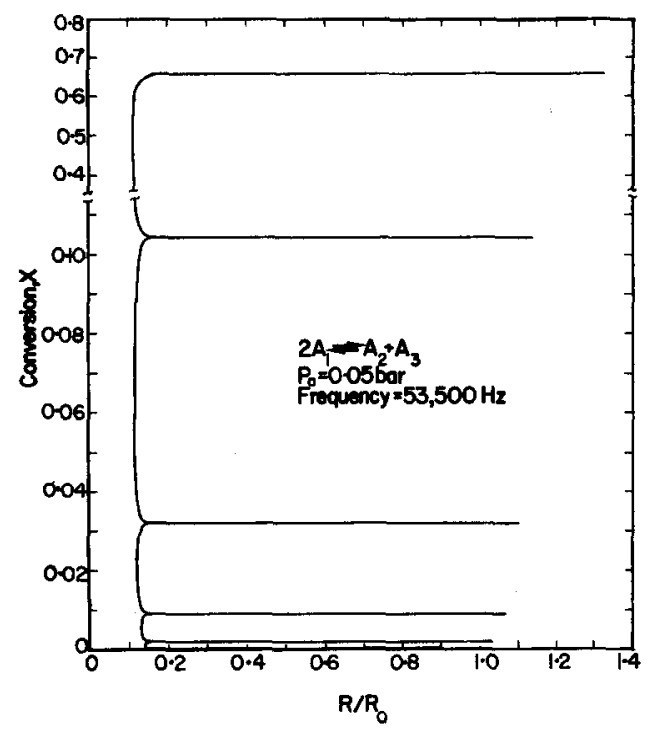

Fig. 10. Conversion-radius curve for a cavity undergoing continuous collapse and rebound.

For this particular set of conditions the conversion reaches its equilibrium value in about five cycles of the cavity oscillation. The lower the activation energy, the fewer will be the number of cycles required for equilibrium conversion to be obtained. 


\section{SUMMARY}

In the preceding analysis, the fluid mechanics of a collapsing cavity and the degree of conversion of the reacting gases in the cavity were studied under a variety of conditions. It was found that the time required to reach a given radius during collapse was greater for an exothermic reaction occurring in the gas phase than for the cavity containing a non-reacting gas. For small heats of reaction, the influence of a reacting gas on the cavity motion is only readily detectable during the rebound of the cavity. When the collapse time approaches the half period of the wave, the conversion increases with increasing acoustic pressure. However, when the collapse time approaches the period of the wave, the conversion first decreases, then increases with increasing acoustic pressure. It was also found that in most instances, 95 per cent of the conversion is accomplished during a 1 per cent change in radius of the collapse. Additional figures and descriptions are presented in the text describing the behavior of the cavity during the collapse and rebound when a gas is reacting in the cavity.

\section{NOTATION}

$A_{i}$ species $i$

$B$ ideal gas constant

$C_{i}$ concentration of species $i, \mathrm{~g}$ moles/cc

$C_{P i}$ heat capacity of species $i, \mathrm{cal} / \mathrm{g}$ mole $/{ }^{\circ} \mathrm{K}$

$E_{a}$ activation energy, cal/g mole

$E$ internal energy, cal

$f_{i}$ frequency factor in Arrhenius rate law

$H$ enthalpy, cal $k_{1}$ forward specific reaction constant

$K$ equilibrium constant

$n_{i}$ number of moles of species $i$, moles

$O_{i}$ order of reaction with respect to species $i$

$P$ pressure, bars or dyn $/ \mathrm{cm}^{2}$

$q$ heat flux out of the cavity, $\mathrm{cal} / \mathrm{sec} / \mathrm{cm}^{2}$

$r$ reaction rate per unit volume, $\mathrm{g}$ mole/ccl sec

$r_{1}$ radial distance from cavity center, $\mathrm{cm}$

$R$ radius of the cavity, $\mathrm{cm}$

$\stackrel{R}{R}$ velocity of the cavity wall, $\mathrm{cm} / \mathrm{sec}$

$\stackrel{R}{R}$ acceleration of the cavity wall, $\mathrm{cm} / \mathrm{sec}^{2}$

$T$ temperature of the gas in the cavity, ${ }^{\circ} \mathrm{K}$

$t$ time, sec

$u$ velocity, $\mathrm{cm} / \mathrm{sec}$

$V$ volume of the cavity, cc

$W$ work done by the cavity on the surroundings, ergs

$X$ moles of species 1 reacted per total mole initially

\section{Greek symbols}

$\tau$ shear stress tensor, $\mathrm{dyn} / \mathrm{cm}^{2}$

$\mu$ shear viscosity, $\mathrm{g} / \mathrm{cm} / \mathrm{sec}$

$\kappa$ dilational viscosity, $\mathrm{g} / \mathrm{cm} / \mathrm{sec}$

$\gamma$ ratio of the specific heats

$\sigma$ surface tension, dyn $/ \mathrm{cm}$

$\rho$ density, $\mathrm{g} / \mathrm{cc}$

$\nu_{i}$ stoichiometric coefficient

$\omega$ angular frequency of the ultrasonic wave, $\sec ^{-1}$

Subscripts

$g$ gas phase

$l$ liquid phase

0 initial condition

[1] RAYLEIGH L., Phil. Mag. 19173494.

\section{REFERENCES}

[2] KIRKWOOD J. G. and BETHE H. A., OSRD Rep. No. 5881942.

[3] TRILLING L., J. appl. Phys. 19522314.

[4] PLESSET M. S. and ZWICK S. A., J. appl. Phys. 19522395.

[5] PLESSET M. S. and HSIEH D. Y., Physics Fluids 19603882.

[6] HICKLING R. and PLESSET M. S., Physics Fluids 1964777.

[7] FLYNN H. G., Physical Acoustics (Edited by W. P. Mason). Academic Press 1964.

[8] NOLTINGK B. E. and NEPPIRAS E. A., Proc. phys. Soc. 1950 B63674.

[9] NOLTINGK B. E. and NEPPIRAS E. A., Proc.phys. Soc. 1951 B64 1032.

[10] GUTH W., Acustica 19566532.

[11] KHOROSHEV G. A., Soviet Phys. Acoust. 19649275.

[12] SCRIVEN L. E., Chem. Engng Sci. 1959101. 


\section{S. FOGLER}

[13] SCRIVEN L. E., Chem. Engng Sci. 19621755.

[14] LIEBERMANN L. N., Phys. Rev. 1949751415.

[15] JAMESON G., Chem, Engng Sci. 19662135.

[16] SMITH J. M., Chemical Engineering Kinetics. McGraw-Hill 1956.

[17] International Critical Tables. (Edited by W. E. Washburn), Vol. 7, pp. 113-173. McGraw-Hill 1930.

[18] Tables of Chemical Kinetics, Homogeneous Reactions. Suppl. 1 1956; Suppl. 2 1960; Suppl. 3 1961. U.S. Department of Commerce, Nat. Bur. Stand. Monogr.

[19] HOUGEN O. A., WATSON K. M. and RAGATZ R. A., Chemical Process Principles, Parts II, III, Wiley 1959.

[20] JANAF Thermochemical Tables. U.S. Department of Commerce, Rep. No. pB-168370. Natn. Bur. Stand. Monogr.

[21] BOGUSLAVSKII Yu Ya, Soviet Phys. Acoust. $1967137,18$.

[22] Handbook of Chemistry and Physics, 39th Edn., (Compiled by C. D. Hodgman). Chemical Rubber Publishing Company 1957.

[23] BULIRSCHER and STOER J., Num. Math. 81.

Résumé- Une analyse a été effectuée sur une cavité en cours d'effondrement contenant des gas de réaction. On a trouvé qu'avec des gaz de réaction dans 'q cavité, l'effondrement d'une cavité contenant des gaz inertes présentait une origine significative dans la mécanique des fluides. En présence de gaz de réaction, il faut résoudre simultanément les équations différentielles non linéaires de mouvement, d'énergie et de cynétique. On a trouvé que le temps nécessaire pour atteindre un rayon donné au cours de l'effol. Irement était plus grand pour une réaction exothermique se produisant en phase gazeuse que pour la cavité contenant un gaz non réactif. Pour la réaction exothermique, le temps d'effondrement s'accroît avec l'ampleur de l'augmentation de chaleur de réaction tandis que pour une réaction endothermique, le temps d'effondrement ne s'accroît pas indéfiniment avec l'augmentation de chaleur de réaction, mais au contraire décroît et se rapproche d'une limite définie. Pour des chaleurs de réaction faibles, il est seulement possible de détecter l'influence d'un gaz réactif sur le mouvement de la cavité au moment du rebondissement de celle-ci. Quand le temps d'effondrement se rapproche de la demi-période de l'onde, la conversion s'accrôt avec l'augmentation de pression accoustique. Toutefois, quand le temps d'effondrement se rapproche de la période de l'onde, la conversion décroît tout d'abord puis accroît avec l'augmentation de pression accoustique. Les descriptions supplémentaires dans le texte tracent le comportement de la cavité pendant l'effondrement quand celle-ci comporte un gaz en cours de réaction.

Zusammenfassung - Es wurde eine Untersuchung über eine, reagierende, Gase enthaltende, zusammenbrechende, Blase durchgefiuhrt. Es konnte festgestellt werden, dass bei Anwesenheit reagierender Gase in der Blase ein deutlicher Unterschied gegenüber der Strömungsmechanik des Zusammenbruchs einer Blase mit inerten Gasen bestand. Bei Anwesenheit reagierender Gase müssen die nichtlinearen Differentialgleichungen der Bewegung, Energie und Kinetik simultan gelöst werden. Es wurde gefunden, dass die Zeit zur Erreichung eines gewissen Radius, während des Zusammenbruchs für eine, sich in der Gasphase abspielende, exotherme Reaktion grösser war als für die Blase mit nichtreagierenden Gasen. Für die exotherme Reaktion erhöht sich die Zusammenbruchszeit mit zunehmender Reaktionswärme, während für eine endotherme Reaktion die Zusammenbruchszeit mit zunehmender Reaktionswärme nicht unbeschränkt zunimmt, sondern sogar abnimmt und einem bestimmten Grenzwert zustrebt. Für geringe Reaktionswärme macht sich die Wirkung eines reagierenden Gases auf die Bewegung der Blase nur während ihres Rückpralls deutlich bemerkbar. Wenn sich die Zusammenbruchszeit der halben Periodendauer einer Welle Nähert, nimmt die Unwandlung mit wachsendem akustischem Druch zu. Nähert sich jedoch die Zusammenbruchszeit der Periodendauer für eine Welle, so fällt die Umwandlung zunächst ab und nimmt dann mit wachsendem akustischem Druck wieder zu. Der Text enthält zusätzliche Beschreibungen, die das Verhalten der Blase während des Zusammenbruchs bei Reaktion eines Gases innerhalb der Blase darlegen. 